\title{
Epidemiología del melanoma en poblacion de la Provincia de Osorno: estudio retrospectivo 2016 - 2018
}

\author{
Cristian Pizarro ${ }^{1}$, Ilonka Hoell ${ }^{1}$, Jovan Araya ${ }^{2}$, Gabriela Araya ${ }^{1}$
}

\section{RESUMEN}

El melanoma maligno ha aumentado su incidencia. En la provincia de Osorno, para el período 2016 2018, se registraron 55 casos nuevos de melanoma, con predominio en sexo femenino. Los subtipos extensivo superficial y nodular fueron los más frecuentes; no obstante el lentiginoso acral fue un grupo prevalente, siendo el sexo masculino el más afectado por esta variedad. Un 49,1\% de los melanomas fueron detectados con Breslow mayor a $1 \mathrm{~mm}$, siendo los hombres los más afectados por una pesquisa más tardía. Reforzar la cobertura de la atención primaria urbana y rural, como así la oferta de dermatólogos, impactarán en la mejora de estos índices.

Palabras claves: melanoma; cáncer de piel; Breslow; epidemiología

\section{SUMMARY}

The Malignant Melanoma has increased its incidence. In the province of Osorno, between 2016-2018 there were 55 new cases of melanoma, with predominance in the female sex. The superficial spreading and nodular subtypes were most common. However the acral lentiginous was a prevalent group, with the male sex being the most affected by this variety. A $49,1 \%$ of melanomas were detected with Breslow greater than $1 \mathrm{~mm}$, with men being the ones who presented later inquiries. The coverage of urban and rural primary care needs to be strengthened, just like supply of dermatologists, it will impact the improvement of these rates.

Key words: melanoma; skin cancer; Breslow; epidemiology

$\mathrm{E}$ 1 melanoma es una neoplasia que deriva de melanocitos epidérmicos. Representa un 4\% de las neoplasias cutáneas ${ }^{1}$ y el $72 \%$ de las muertes por cáncer de piel. ${ }^{2}$

Los melanomas se clasifican en 4 subtipos clínico-histológicos clásicos: de extensión superficial $(70 \%)$, nodular $(15 \%)$, léntigo maligno $(5 \%)$ y acral (5-10\%). Además existen otros subtipos muy poco frecuentes: desmoplásico, nevoide, persistente, entre otros. El principal factor pronóstico de los melanomas es el grosor del tumor (Breslow) ${ }^{3}$

La incidencia de melanoma ha aumentado desde 1970, sobretodo en personas de fototipo claro. ${ }^{4}$

A nivel mundial, la Organización mundial de la salud estimó que el año 2012 hubo una incidencia de 1.7 casos por cada 100.000 habitantes y una tasa de incidencia estandarizada por población mundial de 3 casos por 100.000 habitantes. Se estimó además para el mismo año, una tasa cruda mortalidad de 0.7 muertes por 100.000 personas (tasa estandarizada de 0.9 muertes por cada 100.000 habitantes). ${ }^{5}$

No existe un registro nacional de cáncer en Chile, por lo que no sabemos las cifras exactas de incidencia de éste. El informe oficial de la OMS indica una tasa cruda de 1,9 por 100.000 habitantes, pero sólo se utilizó un registro de Valdivia. ${ }^{5}$

En Osorno no existe registro de los casos de melanoma. Sólo existe un estudio descriptivo de serie de 20 pacientes entre los años 2006-2011. ${ }^{6}$ El objetivo de este trabajo es describir los casos de melanoma entre los años 2016 y 2018 en la Provincia de Osorno y así poder enfocar los recursos hacia áreas más importantes. 


\section{Materiales Y MÉTOdoS}

Se incorporaron los pacientes diagnosticados con melanoma en la red pública del Servicio de Salud Osorno y por dermatólogos del sistema privado, confirmados con biopsia, entre los años 2016 y 2018 en la provincia de Osorno. Seis casos se originaron en el sistema privado, 49 en la red pública. Se consideraron los casos primarios de melanomas y los segundos primarios en pacientes con diagnóstico previo. Se excluyeron de la muestra: metástasis, segundas biopsias y ampliaciones. Los datos recopilados consistieron en edad, sexo, ubicación anatómica, subtipo histológico y Breslow de la lesión. Los datos fueron procesados en programa Excel y el análisis estadístico fue realizado mediante xi cuadrado para muestras pareadas.

\section{Resultados}

Un total de 55 pacientes fueron incluídos en nuestro estudio. 25 de ellos $(45 \%)$ de sexo masculino y $30(55 \%)$ de sexo femenino. La edad promedio de diagnóstico fue de 62,5 + 14,8 años (rango $31-89$ años). No hubo diferencias en la edad de presentación entre ambos sexos (mujeres $61,4+14,9$ y hombres $63,9+14,8$ años). 5 pacientes $(9,0 \%)$ fueron de etnia mapuche en esta muestra.

Respecto al subtipo histológico de la lesión, se encontró que el más frecuente fue el extensivo superficial con 26 casos (47,3\%), seguido del nodular en 10 pacientes (18,2\%). El tercero en frecuencia fue el melanoma lentiginoso acral con 8 casos $(14,5 \%)$. Se encontraron 4 casos $(7,3 \%)$ de otros tipos de melanomas: conjuntival, desmoplásico, indiferenciado y no clasificable. $\mathrm{Al}$ analizar la distribución por sexo, el melanoma extensivo superficial fue el más frecuente en ambos sexos (40,0\% en masculino, y 53,3\% en mujeres), seguido por el subtipo nodular en mujeres $(16,6 \%)$. En hombres, la frecuencia de melanoma lentiginoso acral fue similar al nodular $(20,0 \%)$, ubicándose en el segundo lugar (Gráfico 1). Un 25\% de los melanomas lentiginosos acrales se obtuvieron de pacientes de origen huilliche.
Respecto a la localización anatómica de la lesión, en la muestra estudiada destaca como sitio más frecuente cabeza y cuello $(n=14,23,6 \%)$, y tronco $(n=14,23,6 \%)$. $\mathrm{Al}$ analizar la ubicación respecto al sexo de los pacientes, destaca que en hombres los sitios más frecuentes de presentación fueron tronco $(\mathrm{n}=9,16,0 \%)$ y acral $(\mathrm{n}=6$, $10,9 \%)$, mientras que en mujeres fueron extremidades $(\mathrm{n}=12,21,8 \%)$ y cabeza y cuello $(\mathrm{n}=7,12,7 \%)$ (Gráfico 2). Los subtipos histológicos más prevalentes en cabeza y cuello fueron extensivo superficial ( 6 casos, 42,8\%), lentigo maligno melanoma ( 4 casos, $28,5 \%$ ), nodular (1 caso, $7,1 \%$ y otros (conjuntival, desmoplásico, inclasificable) 3 casos, $21,6 \%$.

$\mathrm{Al}$ analizar el espesor de las muestras obtenidas, 25 pacientes $(45,5 \%)$ presentaron un Breslow menor a $1 \mathrm{~mm}$, 27 pacientes (49,1\%) Breslow igual o mayor a $1 \mathrm{~mm}$, y 3 pacientes presentaron este parámetro inclasificable por las características del tumor (Gráfico 3). Los pacientes cuyo origen es la red pública obtuvieron un Breslow de 2,59 $\mathrm{mm}$, versus práctica privada $1,54 \mathrm{~mm}(\mathrm{p}<0,05)$. Respecto al sexo de los pacientes, destacó un Breslow menor a $1 \mathrm{~mm}$ en un $53,3 \%$ de las mujeres $(n=16)$, versus $36,0 \%$ en hombres $(n=9, p<0,05)$.

\section{Discusión}

La provincia de Osorno tiene una población de 234.122 habitantes según censo 2017, compuesta por 7 comunas, muchas de las cuales presentan altos índices de pobreza y bajo índice de desarrollo humano. ${ }^{7}$ Destaca un mestizaje predominante, con presencia de población huilliche (9,0\% en esta muestra), y caucásica (alemana, austríaca) en menor proporción.

Nuestra serie estudiada está compuesta por 55 pacientes con diagnóstico de melanoma en el trienio 2016 - 2018, un $85,5 \%$ son pacientes atendidos en la red pública. De acuerdo a los datos expuestos, la tasa de incidencia acumulada para este período en nuestra población fue de 23,5 casos por 100.000 habitantes, considerando el período abarcado y la población de la provincia. 


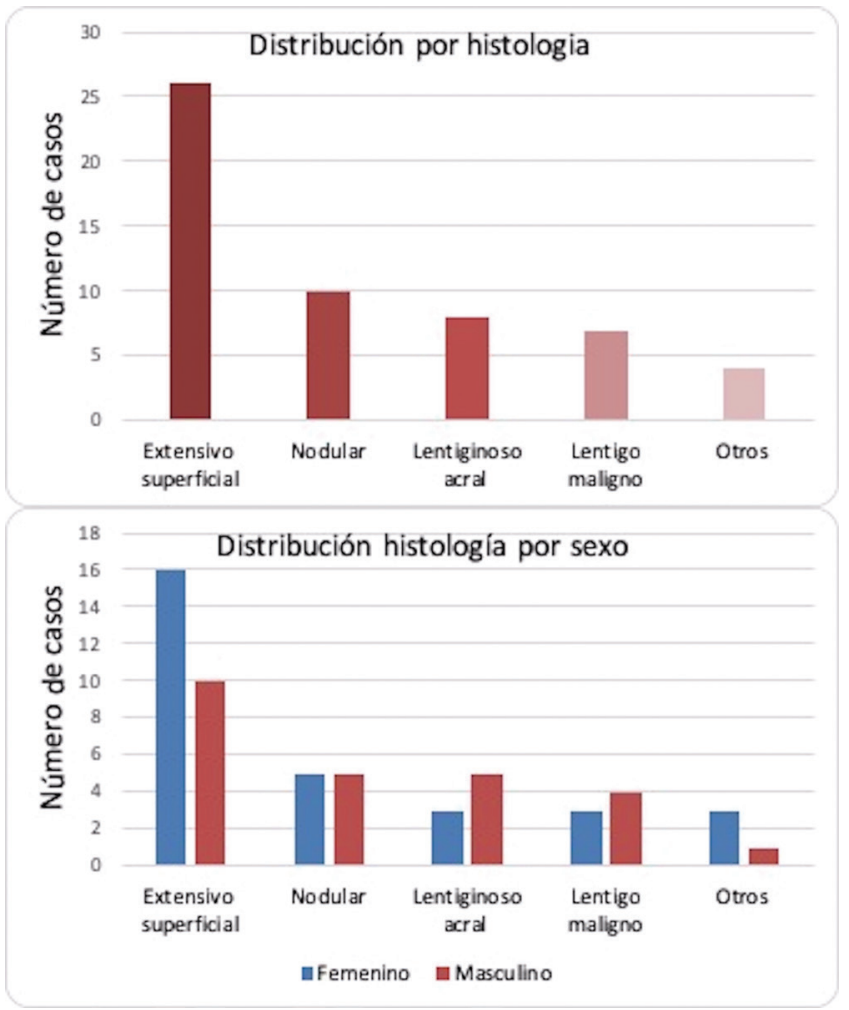

Gráfico 1

Distribución subtipo histológico general y agrupado por sexo

El 2016 se detectaron 12 casos de melanoma, 22 el 2017 y 21 el 2018, lo que coincide con la llegada de más especialistas a la provincia. Esto podría haber influido en el mayor número de casos pesquisados. La incidencia anual fue de 5,12 casos/100.000 hab el 2016; de 9,39 casos / 100.000 el 2017, y de 8,96 casos/100.000 el 2018. Existe fluidez en la derivación de casos cuando el especialista no pertenece a la red pública, y trasferencia a ésta cuando el dermatólogo sí participa en actividad hospitalaria. Nuestra muestra contempla un número importante de casos tanto del área pública como privada si se compara con otros estudios nacionales a nivel provincial, distintos a la región Metropolitana, por lo que nuestros hallazgos pueden servir como referente para otros estudios. ${ }^{6,8}$

Con respecto a la edad, se tiene una media de 62 años, con un rango de 31 a 89 años, lo que coincide con lo reportado en estudio previo en la zona y no difiere respecto a la realidad nacional. ${ }^{6,10}$ La media de edad para pacientes del sector privado fue 10 años menos que para el sector público (51 vs 62 años). Respecto a la distribución por sexo, nuestros datos no difieren con otros estu-

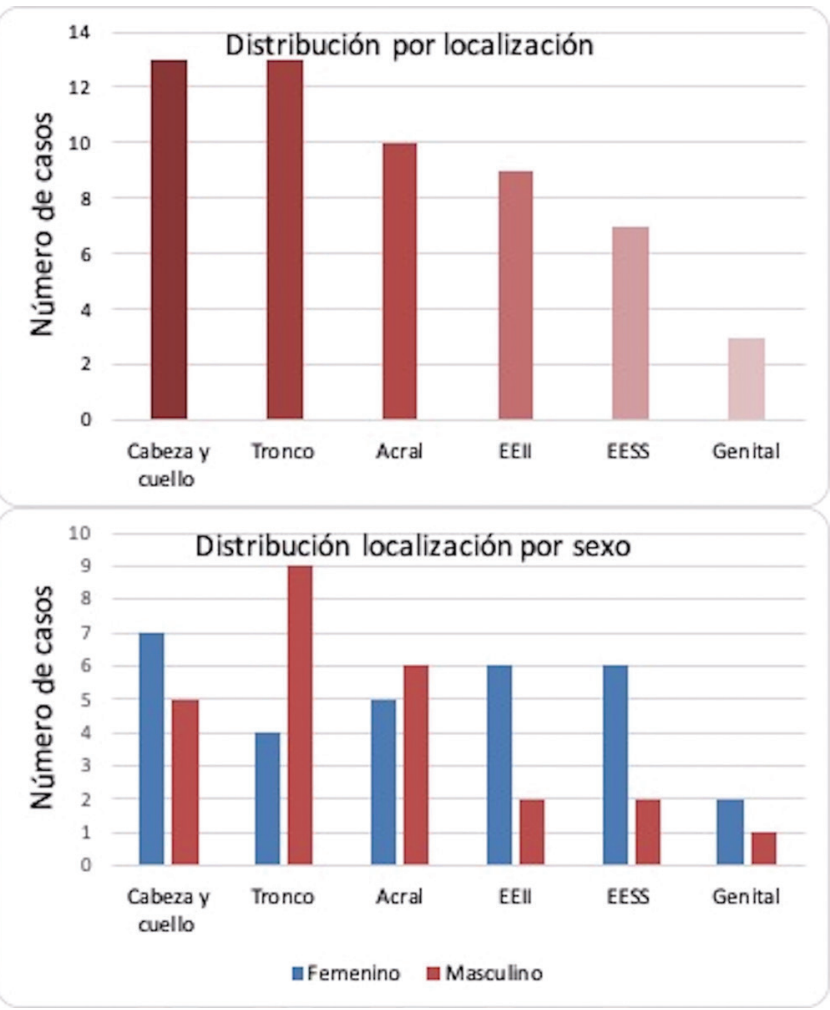

Gráfico 2

Localización de melanomas en grupo estudiado y por sexo

dios nacionales, siendo más frecuente el diagnóstico en la población femenina $(55 \%) \cdot{ }^{10}$

En relación a la distribución anatómica, los melanomas en cabeza, cuello y tronco concentraron el $47 \%$ de los casos. 10 casos de melanoma se presentaron en territorio acral (palmas, plantas y uñas). ${ }^{11,12}$ En sexo femenino predominaron las ubicaciones en cabeza-cuello y extremidades superiores e inferiores, en sintonía otros reportes que señalan estos sitios. ${ }^{10}$ En sexo masculino, las ubicaciones más frecuentes fueron tronco, seguido por territorio acral, lo que refuerza el énfasis del examen físico completo de los pacientes a nivel de médicos en formación y de atención primaria, y especialistas.

Respecto a los subtipos de melanoma, nuestro estudio reporta que el tipo más frecuente fue el extensivo superficial $(47,3 \%)$, seguido por nodular $(18,2 \%)$ y en tercer lugar melanoma lentiginoso acral (14,5\%). Si bien la distribución general coincide con la reportada en la literatura nacional e internacional, el porcentaje de casos de MM extensivo superficial fue menor y el MM lentiginoso acral fue mayor. Los pacientes con lentigo maligno melanoma, al igual que en otras series, presentan un prome- 


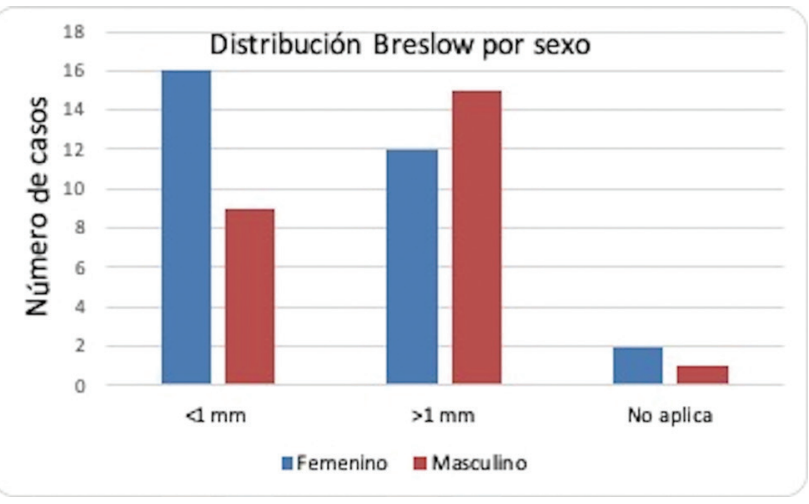

Gráfico 3

Breslow por sexo de grupo estudiado

dio más alto de edad al diagnóstico (en nuestro caso, de $71,1+10,5$ años). A nivel mundial, Fitzpatrick describe entre 2 y $8 \%$ de melanoma lentiginoso acral, Bolognia $4 \%$, y otros autores en publicaciones nacionales como el registro de melanomas de Rancagua, un 2\%., ${ }^{8,13,14}$ Datos de una gran serie de melanomas acrales del gran Santiago mostraron un mayor Breslow en estos tipos de tumores, repitiéndose la tendencia a mayor ubicación plantar en hombres de estratos socioeconómicos bajos y mayor frecuencia de subtipo acral, lo que coincide con nuestro estudio. ${ }^{15,16}$

Respecto a esto último, si bien no hay predominancia étnica en el origen de este subtipo de melanoma (25\% origen huilliche), se destaca que un $87,5 \%$ de los melanomas lentiginosos acrales fueron detectados en la red pública, versus $12,5 \%$ en el sistema privado.

El diagnóstico de melanoma se realizó con Breslow mayor $1 \mathrm{~mm}$ en cerca de un $55 \%$ de los pacientes, si se desglosa por sexo esto alcanzó a un 64\% en el caso de los varones, con una media de edad de 68 años. La amputación de ortejos o dedos impacta importantemente en la fuerza laboral masculina y en la calidad de vida, puesto que a esta edad muchos hombres son aún económicamente productivos.

De los otros casos, uno se presentó como tumor maligno indiferenciado en una pierna (paciente masculino), siendo la inmunohistoquímica la que estableció el diagnóstico, lo que está sugerido en la literatura. ${ }^{17}$ Otras presentaciones fueron vulvar y conjuntival.

Un reciente reporte mostró que niveles bajos de la proteína de unión de vitamina $\mathrm{D}$ puede ser un nuevo factor de riesgo para melanoma, debido a su rol en la activa- ción de los macrófagos contra células tumorales. ${ }^{18}$ Debido a la latitud sur en la que se ubica nuestra provincia, estas mediciones toman relevancia y son un aporte para dirigir nuevos análisis, ya que la intensidad de radiación podría determinar distintos patrones de exposición y presentación de melanomas a lo largo del país. ${ }^{19}$

\section{Conclusión}

La información obtenida a lo largo de este estudio permite comparar nuestra realidad local con la nacional. Siendo una muestra muy limitada es el comienzo del

registro de la realidad local, que será útil para evaluar nuestra labor y generar mejoras.

Es imperioso que los servicios de salud implementen registros de melanomas que permitan generar estadísticas comparables, crear conciencia y visibilidad de estos diagnósticos, de modo de adoptar las mejores políticas públicas que redunden en la calidad de vida de nuestros pacientes. 


\section{REFERENCIAS}

1. Gordon R. Skin Cancer: An Overview of Epidemiology and Risk Factors. Seminars in Oncology Nursing, 2013;29(3): 160-169. https://doi.org/10.1016/j.soncn.2013.06.002

2. Siegel R, Miller K, Jemal A. Cancer statistics, 2017. CA: A Cancer Journal for Clinicians, 2017;67(1):7-30. https://doi.org/10.3322/ caac. 21387

3. Scolyer R, Long G, Thompson J. Evolving concepts in melanoma classification and their relevance to multidisciplinary melanoma patient care. Molecular Oncology 2011;5(2): 124-136. https://doi. org/10.1016/j.molonc.2011.03.002

4. International Agency for Research on Cancer, WHO. CI5: Cancer incidence in five continents. 2014

5. Ferlay J, Soerjomataram I, Dikshit R, Eser S, et al. Cancer incidence and mortality worldwide: Sources, methods and major patterns in GLOBOCAN 2012. International Journal of Cancer, 2014;136(5):359-386. https://doi.org/10.1002/ijc.29210

6. Leigh S, Zarges P, Contreras T, Jans $\mathrm{J}$ et al. Perfil clínico y epidemiológico de una serie consecutiva de pacientes con diagnóstico de melanoma cutáneo en la provincia de Osorno: Período 2006-201 1. Cuadernos de Cirugía 2011;25(1):37-42. https://doi.org/10.4206/ cuad.cir.2011.v25n1-06

7. Instituto Nacional de Estadística de Chile. Población total por sexo y área urbana-rural. Estadísticas de la Región de Los Lagos. Gobierno de Chile 2019. Disponible en: https://regiones.ine.cl/los-lagos/ estadisticas\#Estadisticas_de_Poblacion [Consultado el 10 de agosto de 2019].

8. Perez, M. E., Bley, C., Cardenas, C., \& Ramírez, C. Epidemiología del melanoma en la Región de O'Higgins durante los años 2012 y 2015. Revista Chilena de Dermatología 2018;33(4):43-48. https:// doi.org/10.31879/rcderm.v33i4.164

9. Kaplan V, Morales C, Bobadilla F, Fernandez J et al. Caracterización epidemiológica e histopatológica de metástasis cutáneas en la población consultante de 2 hospitales de Santiago durante los años 2005 a 2017. Actas Dermosifiliogr. 2018. https://doi.org/10.1016/j. ad.2018.07.012

10. Zemelman V, Araya I, Valenzuela C. Medina M, et al. Melanoma maligno en población pública de Santiago, Chile: correlación entre localización anatómica, genero y edad. Rev Chilena Dermatol. 2009;25(4):333-38

11. Durbec F, Martin L, Derancourt C, Grange F. Melanoma of the hand and foot: epidemiological, prognostic and genetic features. A systematic review. British Journal of Dermatology 2012;166(4):727739. https://doi.org/10.1111/j.1365-2133.2011.10772.x

12. Piliang P. Acral lentiginous melanoma. Surgical Pathology Clinics 2012;2(3):535-541. https://doi.org/10.1016/j.path.2009.08.005

13. Bailey E. "Melanoma cutáneo". En: Fitzpatrick Dermatología en Medicina General. Goldsmith, Katz Gilchret, Paller, Leffell, Wolff, Editores. 8va edición. Editorial Médica Panamericana; 2014; p 1416-1444

14. Garbe C. "Melanoma". En: Dermatology. Third Edition. Bolognia, Jorizzo, Schaffer, Editores. Elsevier Saunders; 2012; 1885 - 1910
15. Zemelman V, Roa J, Ruiz Tagle S, Valenzuela C. Malignant melanoma in Chile: an unusual distribution of primary sites in men from low socioeconomic strata. Clinical and Experimental Dermatology 2006;31(3):335-338. https://doi.org/10.1111/ j.1365-2230.2005.02038.x

16. Zemelman V, Yagnam M, Araya I, Valenzuela F, et al. Melanoma maligno acral en Chile: análisis clínico e histopatológico de 70 casos en la población consultante de hospitales públicos en Santiago, Chile. Rev. Chilena Dermatol. 2015;31(2): 141 - 144

17. Cabrera R, Recule F. Unusual clinical presentatiosn of malignant melanoma: a review of clinical and histologic features with special emphasis on dermatoscopic findings. Am $\mathrm{J}$ of Clin Dermatol 2018;19(1):S15-S23

18. Navarrete-Dechent C, Del Puerto C, Molgó M, González S, et al. Circulating vitamin D-binding protein and free 25-hydroxyvitamin $\mathrm{D}$ concentrations in patients with melanoma: A case-control study. Journal of the American Academy of Dermatology 2017;77(3):575577. https://doi.org/10.1016/j.jaad.2017.03.035

19. Rivas M, Rojas E, Calaf M., Barberán M, et al. Association between non-melanoma and melanoma skin cancer rates, vitamin $\mathrm{D}$ and latitude. Oncology Letters 2017;13(5):3787-3792. https://doi. org/10.3892/ol.2017.5898 